\title{
Yes, Breast Surgeons May Provide Breast Cancer Genetic Assessment and Testing
}

\author{
Cary S. Kaufman, MD, FACS \\ Department of Surgery, University of Washington, Bellingham Regional Breast Center, Bellingham, WA
}

The article by Beitsch and Whitworth is particularly timely for several reasons. ${ }^{1}$ There is underutilization of genetic testing and counseling to identify breast cancer mutation carriers before they develop breast cancer; the next generation of genetic testing has arrived with the ability to test for multiple mutations simultaneously; some payers have instituted severe restrictions on any genetic testing unless the payer's genetics professionals have approved the test; and although there are no standard credentialing criteria for the various providers of breast cancer genetic testing and counseling, there are defined guidelines from credible national societies.

The limited availability of risk assessment, genetic testing and counseling services has several causes. Certified genetic counselors can provide these services, but there are not enough genetic counselors to service the existing population of breast cancer patients, let alone the high-risk undiagnosed patients. There are about 3,000 certified genetics counselors who provide services across the county. ${ }^{2}$ Many are in populated states and big cities (e.g., 46 in Pennsylvania who deal with cancer, 12 located within 2 miles of each other in Philadelphia), with many cities and some states left without face-to-face access with a certified genetics counselor (e.g., only three in the State of Alaska, all three located in Anchorage, and none in the entire state of Wyoming). ${ }^{3}$ Most patients who are identified with breast cancer mutations are identified only after they have developed cancer. The most common mutation is the hereditary breast cancer and ovarian cancer syndrome (HBOC). The goal should be to identify mutation carriers before they develop breast cancer. Such a

\footnotetext{
(C) Society of Surgical Oncology 2014
}

First Received: 9 September 2014;

Published Online: 8 October 2014

C. S. Kaufman, MD, FACS

e-mail: breastcare@aol.com screening system is present in some breast centers with an integrated risk assessment questionnaire within the registration papers for routine screening mammography. ${ }^{4}$ As mentioned by Beitsch and Whitworth, there is inadequate identification of women before their breast cancer diagnosis, underscoring the need for risk assessment by clinicians. ${ }^{5}$

This is a unique time in the field of genetics and the identification of cancer causing genetic mutations. The next generation mutation identification technology has afforded the ability to test for multiple genetic mutations beyond HBOC syndrome simultaneously. This provides the patient a better opportunity to be identified as a mutation carrier and appropriately counseled. The expense of single site genetic testing has prohibited many patients with strong breast cancer family histories and negative results HBOC from affording a second site genetic mutation test. With the new technology, test panels for multiple breast cancer mutations can be processed simultaneously providing a comprehensive assessment for the presence of a breast cancer genetic mutation. Early studies using a 25 -gene mutation panel have shown the number of pathologic gene mutations identified can be increased by as much as $51 \%$. $^{6}$

Guidelines for risk assessment and genetic counseling and testing are well established and have been documented by national organizations. Beitsch and Whitworth comment that the National Comprehensive Cancer Network (NCCN) guidelines provide criteria to identify candidates for genetic counseling and mutation testing. The guidelines also describe the health care provider who would offer such counseling and testing: "A genetic counselor, medical geneticist, oncologist, surgeon, oncology nurse, or other health professional with expertise and experience in cancer genetics should be involved early in counseling patients who potentially meet criteria for an inherited syndrome." 7 The NCCN guidelines do not identify the specific health care degree required or the specific education and experience for this individual. 
The National Accreditation Program for Breast Centers (NAPBC) has representatives from 20 professional organizations dealing with breast cancer including oncologists, surgeons, and genetic counselors. The NAPBC board has spent many hours over many months discussing the issues of risk assessment, genetic counseling, and genetic testing. A structured approach defining pretest and posttest counseling has been developed in a Genetics Standard of Care for Breast Centers. Several types of breast care professionals are described who can provide these services in a breast center. ${ }^{8}$ According to the NAPBC, the description for a physician qualified to provide these services includes a "physician or other trained health care professional with expertise and experience in cancer genetics (defined as providing cancer risk assessment on a regular basis)." These statements from the NCCN and NAPBC were not developed as a result of light conversation. Thorough discussions and debate preceded any final standard or guideline based on vetting from varied medical opinions.

It may be understandable that some payers wish to curtail payments for genetic testing when there is suggestion that inappropriate testing has occurred. Patients whose family history does not justify genetic testing are sometimes tested. This is an overuse of genetic testing and should be avoided. Yet instances of inappropriate testing are not an adequate reason to stop testing by all providers but rather a reason to confirm that individual providers conform to established guidelines for counseling and testing. When payers limit testing by all providers, identification of existing mutation carriers will decrease and patient care will suffer.

The topic of genetic testing was discussed by the American Medical Association in June 2014, and after thorough discussion, an AMA resolution was developed. Resolution 115 opposes limiting the ordering of genetic testing based solely on physician specialty, and it opposes the utilization of nonaffiliated medical specialists or nonphysicians before ordering genetic testing. ${ }^{9}$ This is a direct response to the unilateral requirements by some payers to ignore the patient's physician recommendation and defer decision to the payers' genetic professional, who is not directly involved with the patient.

One reason why payers restrict testing is because of the expense of genetic testing. If genetic testing cost the same as a routine chemistry panel, there would be no added barrier to genetic testing. Some payers do not seem to trust a breast surgeons' ability to make an accurate decision regarding genetic testing. Yet breast surgeons are commonly making major medical decisions that have a significant financial impact. Breast surgeons make decisions regarding operations and reoperations, reconstruction, the 21-gene assay, postoperative radiation, intraoperative radiation or brachytherapy, and so on. Like these other decisions, genetic assessment and testing require clinical assessment according to guidelines and medical judgement.

This survey conducted by the ASBS was fruitful but has some limitations. It was fruitful in that it gave us a perspective on the self-assessment of a large body of breast surgeons from different practice patterns across the country. For those that were providing genetic counseling and testing, a picture of appropriate clinical care emerged from the survey. In addition, many breast surgeons expressed a willingness to learn more about providing these genetic services in the future.

One of the limitations of this survey is that it is based on self-assessment. No confirmation was provided that these self-assessments were accurate or true. Do surgeons actually provide both the pre- and posttest counseling, as they claim? Is it accurately self-reported or just hopeful thinking? I suspect that it is accurate because the majority of breast surgeons in the ASBS are serious about their profession and have supported previous surveys of the ASBS with accurate data. Another nominal limitation is that 900 people responded from a total of over 2,600 surveys (35\%). Although this is less than half, it is a healthy response rate. This population of breast surgeons are interested in this topic and report that they provide appropriate care.

There is an increased need to identify women who carry a breast cancer mutation, preferably before their diagnosis. Breast surgeons are positioned to see these patients early in their care journey and routinely obtain a family history. It is unfortunate that some insurance companies have acted to obstruct genetic counseling and testing rather than to facilitate adherence to established guidelines and standards for genetic counseling and testing. Guidelines from the NCCN and standards from the NAPBC provide the quality foundation for this clinical field. Beyond complying with these standards, breast surgeons are interested in further education in this dynamic field. At the dawn of nextgeneration technology that may identify more mutation carriers, I would hope that insurance companies do not try to prevent the inevitable future from occurring. It is futile to create barriers to appropriate testing.

ACKNOWLEDGMENT The author declares no conflict of interest.

\section{REFERENCES}

1. Beitsch P, Whitworth P. Can breast surgeons provide breast cancer genetic testing? An American Society of Breast Surgeons survey. Ann Surg Oncol. In press.

2. National Society of Genetics Counselors. http://nsgc.org/p/cm/ld/ fid=7. Accessed Sep 4, 2014.

3. National Society of Genetics Counselors. http://nsgc.org/p/cm/ld/ fid=164. Accessed Sep 4, 2014. 
4. Brinton JT, Barke LD, Freivogel ME, Jackson S, O'Donnell CI, Glueck DH. Breast cancer risk assessment in 64,659 women at a single high-volume mammography clinic. Acad Radiol. 2012;19:95-9.

5. Drohan B, Roche CA, Cusack JC, Hughes KS. Hereditary breast and ovarian cancer and other hereditary syndromes. Ann Surg Oncol. 2012;19:1732-7.

6. Allen B, Tung N, Batelli C, et al. Prevalence of gene mutations among hereditary breast and ovarian cancer patients using a 25 gene panel. Paper presented at: American College of Medical Genetics annual meeting, March 2014. http://ww2.aievolution. com/acm1401/index.cfm?do=abs. viewAbs\&abs=2176.

7. NCCN Clinical Practice Guidelines in Oncology. Genetic/familial high-risk assessment: breast and ovarian. Version 1.2014. http:// www.nccn.org/professionals/physician_gls/pdf/genetics_screening. pdf. Accessed Sep 4, 2014.

8. NAPBC Standards Manual page 54-55 found at: https://www.facs. org/ /media/files/quality\%20programs/napbc/2014\%20napbc\%20 standards\%20manual.ashx. Accessed Sep 6, 2014.

9. American Medical Association. June meeting. http://www.mag. org/sites/default/files/downloads/ama14-refcommA-report.pdf. Accessed Sep 4, 2014. Found at: https://www.ssl3.amaassn.org/ apps/ecomm/PolicyFinderForm.pl?site=www.amaassn.org \&uri $=\%$ 2 fresources $\% 2 \mathrm{fhtml} \% 2 \mathrm{fPolicy} F i n d e r \% 2$ fpolicyfiles $\% 2 \mathrm{fHnE} \% 2 \mathrm{fH}-$ 460.902.HTM. Accessed Oct 6, 2014. 